\title{
TOXICIDADE AGUDA E CRÔNICA DO EXTRATO DE NIM (Azadirachta indica) PARA Ceriodaphnia dubia
}

\author{
RAFAEL GROSSI BOTELHO* \\ MARÍLIA MITIE INAFUKU ** \\ LUCINEIDEAPARECIDA MARANHO* \\ LUÍS MACHADO NETO*** \\ RICARDO ALVES DE OLINDA**** \\ CARLOS TADEU DIAS $* * * * *$ \\ VALDEMAR LUÍS TORNISIELO*****
}

\begin{abstract}
A preocupação com as questões ambientais tem levado ao crescimento de pesquisas relacionadas aos compostos com potencial no controle de pragas na agricultura, direcionando alternativas que minimizem os impactos ao ambiente. Nesse sentido acredita-se que a utilização de produtos proveniente de plantas possa representar opção eficaz para o controle de pragas com menor risco ambiental. Objetivou-se com este trabalho avaliar a toxicidade aguda e crônica de uma formulação comercial de nim para Ceriodaphnia dubia. A CE CE $_{50}(48 \mathrm{~h})$ determinada foi de $0,032 \mathrm{~mL} \cdot \mathrm{L}^{-1}$, e os valores de CENO, CEO e VC foram respectivamente em $\mathrm{mL} \cdot \mathrm{L}^{-1}$ : 0,$004 ; 0,008$ e 0,006 . Os testes de toxicidade mostraram efeitos do produto tanto na mobilidade como na reprodução de C. dubia.
\end{abstract}

PALAVRAS-CHAVE: Ceriodaphnia dúbia; $\mathrm{CE}_{50}$; NIM.

* Doutorandos em Química na Agricultura e no Ambiente, Centro de Energia Nuclear na Agricultura (CENA) Universidades São Paulo (USP), Piracicaba, SP (e-mail: rbotelho@cena.usp.br;Imaranho@cena.usp.br).

** Mestranda em Química na Agricultura e no Ambiente, CENA/USP Piracicaba, SP (e-mail: mminafuku@cena.usp.br).

*** Tecnólogo em Biocombustíveis pela Faculdade de Tecnologia de Piracicaba (FATEC), Piracicaba, SP (e-mail: Imachado@cena.usp.br).

**** Doutorando em Estatística e Experimentação Agronômica pela Escola Superior de Agricultura Luiz de Queiroz (ESALQ/USP), Piracicaba, SP (e-mail: ricardoestatistico@usp.br).

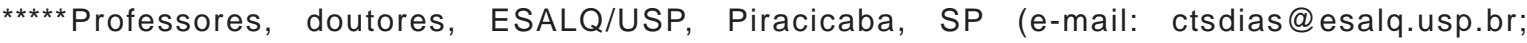
vitornis@cena.usp.br). 


\section{INTRODUÇÃO}

Desde a Revolução Industrial, a agricultura se destaca como a maior responsável pela perda de biodiversidade, principalmente em ambientes aquáticos, já que a utilização de agrotóxicos para o controle de pragas acaba por contaminar também os ambientes aquáticos (EDWIN, 1996).

Dentre as classes dos agrotóxicos existentes, os inseticidas são extremamente utilizados no Brasil para garantir a produtividade das culturas apesar de sua alta toxicidade ambiental. A alternativa para minimizar danos ao ambiente tem sido a utilização de extratos derivados de plantas, pois acreditase que essas substâncias apresentam a mesma eficiência no controle de pragas que os inseticidas sintéticos e causam menor impacto ambiental (COSTA, SILVA e FIUZA, 2004; CRUZ, MACHADONETO e MENEZES, 2004).

O Nim (Azadirachta indica), pertencente à família Meliácea, é originário da Índia. Tem sido usado na produção de madeira, como planta medicinal, e mais recentemente como bioinseticida. Extratos à base de nim causam inibição da alimentação dos insetos, afetando o desenvolvimento das larvas, reduzindo a fecundidade e a fertilidade dos adultos (REMBOLD, 1989; RADCLIFFE et al., 1991; MARTINEZ, 2002).

Apesar de serem utilizados na agricultura, dependendo da maneira de aplicação, os inseticidas podem contaminar ambientes aquáticos, e, por isso, é extremamente importante a utilização de testes de toxicidade de produtos derivados de plantas para organismos aquáticos (DUNKEL e RICHARDS, 1998; GOKTEPE e PLHAK, 2002; CRUZ, MACHADO-NETO e MENEZES, 2004). Desta forma, objetivouse com este trabalho, avaliar a toxicidade aguda e crônica de uma formulação comercial à base de nim sobre o microcrustáceo Ceriodaphnia dubia.

\section{MATERIAL E MÉTODOS}

\subsection{MANUTENÇÃO E CULTIVO DE Ceriodaphnia dubia}

Para a manutenção do cultivo de $C$. dubia seguiram-se as normas estabelecidas pela ABNT (2005). Os organismos foram mantidos em frascos de vidro contendo 1,5 litros de meio MS (KEATING, 1985) e 70 organismos adultos em cada cultura. Os frascos foram mantidos em incubadora com temperatura entre 25 a $28^{\circ} \mathrm{C}$ e luminosidade de 16 horas luz e 8 horas escuro. A renovação do meio de cultivo ocorreu preferencialmente duas vezes por semana. Para alimentação dos indivíduos foi fornecido alga $P$. subcapitata (aproximadamente $1 \times 10^{5}$ células por organismo) três vezes por semana.

\subsection{TESTE DE TOXICIDADE AGUDA}

Para a execução do teste de toxicidade aguda seguiram-se as normas da ABNT (2004). Realizou-se teste preliminar para determinar as concentrações a serem utilizadas neste estudo. No teste definitivo, foram utilizadas quatro concentrações do produto comercial Bioneen além do grupo controle, cada uma com duas réplicas. Em cada réplica foram adicionados cinco organismos neonatos com idade entre 6 a 24 horas. Primeiramente, preparou-se a solução mãe de $1 \mathrm{~mL} \cdot \mathrm{L}^{-1} \mathrm{do}$ produto e a partir dessa, soluções com outras concentrações para serem utilizadas no teste. Para o preparo das diluições utilizou-se meio de cultivo MS e as seguintes concentrações em $\mathrm{mL} \cdot \mathrm{L}^{-1}: 0,01 ; 0,1 ; 1,0$ e 10,0 . As soluções teste foram colocadas em frascos de vidro transparente com capacidade de $20 \mathrm{~mL}$, sendo posteriormente adicionados os organismos com auxílio de pipeta Pasteur. Para alimentação dos organismos utilizou-se alga $P$. subcapitata, sendo os frascos teste incubados nas mesmas condições do cultivo. Após 48 horas de exposição determinou-se a Concentração Efetiva Média $\left(\mathrm{CE}_{50}\right)$. 


\subsection{TESTE DE TOXICIDADE CRÔNICA}

Para a execução do teste de toxicidade crônica seguiram-se as normas da ABNT (2005). A partir do valor de $\mathrm{CE}_{50}$ (48h) foram estabelecidas concentrações utilizando-se o fator de diluição 0,5. Nesse teste, foram usadas cinco concentrações do produto e um grupo controle com 10 réplicas cada, contendo um organismo com idade entre 6 a 24 horas. Assim como no teste de toxicidade aguda, preparou-se a solução mãe de $1 \mathrm{~mL} \cdot \mathrm{L}^{-1}$ do produto e a partir dessa foram efetuadas as demais concentrações. As soluções teste utilizadas foram em mL.L-1: 0,001; 0,002; 0,004; 0,008 e 0,016 . As condições do teste crônico foram iguais à do teste agudo, no entanto, com tempo de exposição de 7 dias, sendo que a cada dois dias as soluções foram renovadas e o organismo adulto transferido para outro recipiente de igual volume. A cada renovação e no final do teste, o número de filhotes de cada frasco foi registrado.

\subsection{ANÁLISE ESTATÍSTICA}

A Concentração Efetiva Média a $50 \%$ dos indivíduos $\left(\mathrm{CE}_{50}\right)$ foi determinada por meio do programa estatístico Trimed Spearman Karber (HAMILTON RUSSO e THURSTON, 1977). Já a Concentração de Efeito Não Observado (CENO) e a Concentração de Efeito Observado (CEO) foram determinadas para o número de neonatos produzidos por fêmea, utilizando-se análise de variância (ANOVA) seguida pelo teste de Dunett's $(a ́=0,05)$ através do programa TOXSTAT versão 3.3.

\section{RESULTADOS E DISCUSSÃO}

$A \mathrm{AE}_{50}(48 \mathrm{~h})$ determinada do produto Bioneen para C. dubia foi $0,032 \mathrm{~mL} \cdot \mathrm{L}^{-1}$. Percebeu-se que ao final de $48 \mathrm{~h}$ de exposição, o controle e a concentração de $0,01 \mathrm{~mL} \cdot \mathrm{L}^{-1}$ não afetaram a mobilidade dos organismos. No entanto, nas concentrações de 0,$1 ; 1$ e $10 \mathrm{~mL} \cdot \mathrm{L}^{-1}$ foi observada $100 \%$ de imobilidade (Figura 1).

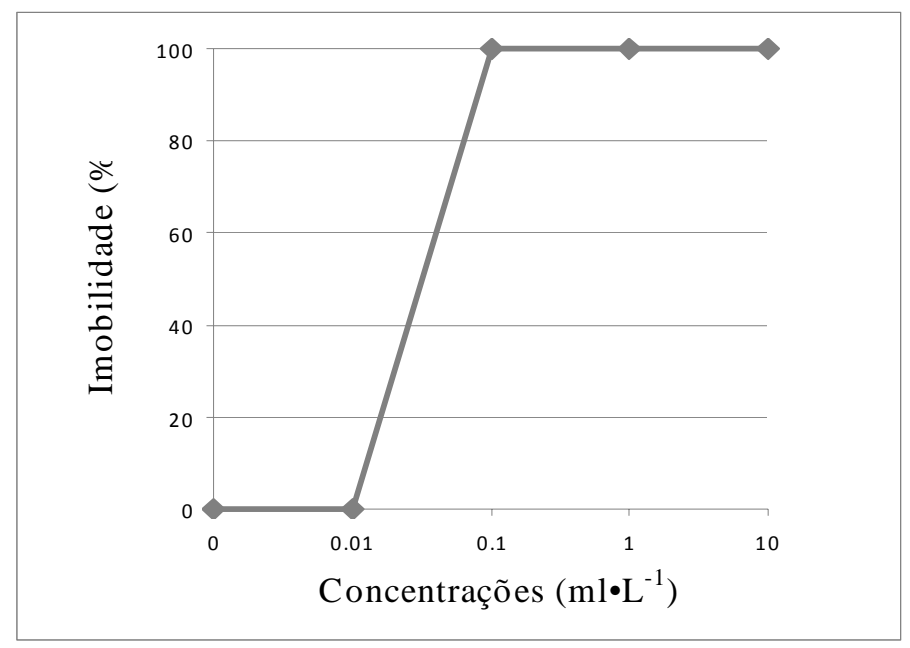

FIGURA 1 - IMOBILIDADE DE C. dubia APÓS 48 HORAS DE EXPOSIÇÃO A DIFERENTES CONCENTRAÇÕES DE BIONEEN 
Em relação ao teste de toxicidade crônica, o aumento das concentrações diminuiu o número de neonatos por fêmea como pode ser observado na Figura 2.

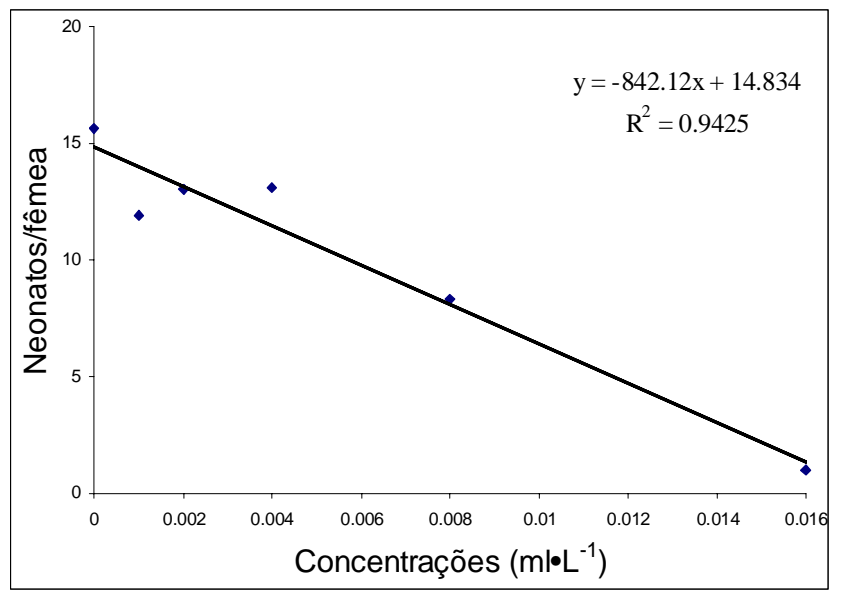

\section{FIGURA 2 - NÚMERO MÉDIO DE NEONATOS/FÊMEA DE C. dúbia APÓS 7 DIAS DE EXPOSIÇÃO A DIFERENTES CONCENTRAÇÕES ( $\left.x^{\star}\right)$ DE BIONEEN}

Ainda em relação à reprodução, os valores de CENO, CEO e VC foram, respectivamente, em $\mathrm{mL} \cdot \mathrm{L}^{-1}$ : 0,004; 0,008 e 0,006 para o organismo testado (Tabela 1). Verificou-se também que os organismos expostos a concentrações de 0,$001 ; 0,002$ e $0,004 \mathrm{~mL} \cdot \mathrm{L}^{-1}$ não diferiram estatisticamente do controle em relação ao número de neonatos produzidos, diferente do que foi observado para as concentrações de 0,008 e $0,016 \mathrm{~mL} \cdot \mathrm{L}^{-1}$ (Tabela1).

Estudos avaliando a toxicidade de diferentes formulações comerciais à base de nim em organismos aquáticos têm sido realizados. SAUCKE e SCHMUTTERER (1992) encontraram valor de $\mathrm{CE}_{50}$ da formulação comercial Margosan-O de 0,19 ppm para Daphnia magna. Nesse mesmo estudo, outras formulações comerciais foram testadas e os valores de $C_{50}$ variaram de 0,04 a 3,38 ppm. SAUCKE e SCHMUTTERER (1992) observaram ainda que a toxicidade de outros produtos formulados de nim foi 10 vezes mais tóxica do que o extrato aquoso de sementes do nim, o que lhes permitiu afirmar que os emulsificantes e outros ingredientes presentes nas formulações comerciais testadas foram as principais causas da toxicidade para $D$. magna.

TABELA 1 - NÚMERO MÉDIO DE NEONATOS/FÊMEA DE C. dúbia APÓS 7 DIAS DE EXPOSIÇÃO ÀS CONCENTRAÇÕES DE BIONEEN

\begin{tabular}{c|c}
\hline Concentrações $\left(\mathbf{m L} \cdot \mathrm{L}^{-\mathbf{1}}\right)$ & Média de reprodução (neonatos/fêmea) \\
\hline 0 & $15.500 \mathrm{a}$ \\
0,001 & $11.900 \mathrm{a}$ \\
0,002 & $13.000 \mathrm{a}$ \\
0,004 & $13.100 \mathrm{a}$ \\
0,008 & $8.300 \mathrm{~b}$ \\
0,016 & $1.000 \mathrm{~b}$ \\
\hline
\end{tabular}

Médias seguidas por letras iguais não diferem entre si pelo teste de Dunnett's com á = 0,05. 
STARK (2001) determinou o valor de $\mathrm{CL}_{50}$ da formulação comercial Nimix para Daphnia pulex em 0,68 ppm. Quando avaliou a toxicidade dessa mesma formulação comercial na reprodução, os valores de CENO e CEO foram respectivamente 0, 045 e 0,15 ppm.

GOKTEPE e PLHAK (2002) avaliaram a toxicidade da formulação comercial Bioneen (a mesma utilizada no presente estudo) em $D$. pulex e obtiveram $0,033 \mathrm{~mL} \cdot \mathrm{L}^{-1}$ como valor de $\mathrm{CL}_{50}$. $\mathrm{O}$ resultado obtido por esses autores ficou próximo da $\mathrm{CE}_{50}$ encontrada no presente estudo, sugerindo sensibilidade semelhante de $D$. pulex e $C$. dúbia a essa formulação comercial.

De acordo com SUTTON (2002), os inseticidas à base de nim podem apresentar riscos ao ambiente aquático, principalmente, aos insetos e zooplâncton (especialmente os copépodas), motivo pelo qual devem ser estudados mais cautelosamente.

\section{CONCLUSÃO}

Os testes de toxicidade aguda e crônica mostraram toxicidade do Bioneen, tanto na mobilidade como na reprodução de $C$. dubia nas maiores concentrações. No entanto, para afirmar que se trata de produto perigoso ao ambiente aquático, bioensaios toxicológicos com outros organismos devem ser realizados. Estudos mais aprofundados também podem ser sugeridos, visando estabelecer protocolos de risco ambiental para compostos de extratos de derivados de plantas.

\section{ABSTRACT \\ ACUTE AND CHRONIC TOXICITY OF THE EXTRACT OF NEEM (Azadirachta indica) TO Ceriodaphnia dubia}

The concern with environmental issues has led to the growth of research on compounds with potential to control pests in agriculture, directing alternatives that minimize the environmental impact. In this sense it is believed that the use of products from plants can be an effective option for pest control with less environmental risk. The objective of this study was to evaluate the acute and chronic toxicity of a commercial formulation of neem to Ceriodaphnia dubia. The $\mathrm{EC}_{50}$ (48h) was determined from $0.032 \mathrm{~mL} \mathrm{~L}^{-1}$, and the values of NOAEL, LOAEL and CV were respectively in $\mathrm{mL} \mathrm{L}^{-1}: 0.004,0.008$ and 0.006 . Toxicity tests showed effects of the product both in stillness and in the reproduction of $C$. dubia.

KEY-WORDS: $E C_{50}$; Ceriodaphnia dubia; NEEM.

\section{REFERÊNCIAS}

1 ABNT. Associação Brasileira de Normas Técnicas. NBR 13373: ecotoxicologia aquática - toxicidade aguda - método de ensaio com Daphnia spp (Crustácea, Cladocera). Rio de Janeiro, 2004. 21 p.

2 ABNT. Associação Brasileira de Normas Técnicas. NBR 13373: ecotoxicologia aquática - toxicidade crônica método de ensaio com Ceriodaphnia spp (Crustacea, Cladocera). Rio de Janeiro, 2005. 15 p.

3 COSTA, E. L. N.; SILVA, R. F. S.; FIUZA, L. M. Efeitos, aplicações e limitações de extratos de plantas inseticidas. Acta Biológica Leopoldensia, São Leopoldo, v. 26, n. 2, p.173-185, 2004.

4 CRUZ, C.; MACHADO-NETO, J. G.; MENEZES, M.L.de. Toxicidade aguda do inseticida paration metílico e do biopesticida azadiractina de folhas de nim (azadirachta indica) para alevino e juvenil de pacu (Piaractus mesopotamicus). Pesticidas: revista de ecotoxicologia e meio ambiente, v.14, p.93-102, 2004.

5 DUNKEL, F.V.; RICHARDS, D.C. Effect of an azadirachtin formulation on six nontarget aquatic macroinvertebrates. Environmental Entomology, v. 27, n.4, p. 667-674, 1998.

6 EDWIN, D.O. Control of water pollution from agriculture. FAO Irrigation and Drainage Paper, v, 55, p. 1-101, 1996.

7 GOKTEPE, I.; PLHAK, L.C. Comparative toxicity of two azadirachtin-based nim pesticides to Daphnia pulex. Environmental Toxicology and Chemistry, v. 21, n. 1, p. 31-36, 2002. 
8 HAMILTON, M. A.; RUSSO, R.C.; THURSTON, R.V. Trimed Spearman-Karber method for estimating median lethal concentration in toxicity bioassays. Environmental Science Technology, v.7, n.11, p.714 -719, 1977.

9 KEATING, K.I. A system of defined (sensu stricto) media for daphnid (Cladocera) culture. Water Research, v. 19, n.1, p. 73-78, 1985.

10 MARTINEZ, S.S. Ação do nim sobre os insetos. In: MARTINEZ, S.S. (ed). O Nim - Azadirachta indica: natureza, usos múltiplos, produção. Londrina: Instituto Agronômico do Paraná, 2002. p. 31-57.

11 RADCLIFFE, E. B.; DUNKEL, F.V.; STRZOK, P.P.; ADAM, S. Antifeedant effect of nim, Azadirachta indica A. Juss., kernel extracts on Kraussaria angulifera (Krauss) (Orthoptera:Acrididae), a Sahelian grasshopper. Tropical Agriculture, v. 68, n.1, p. 95-101, 1991.

12 REMBOLD, H. Isomeric azadirachtins and their mode of action. In: JACOBSON, M. (ed). Focus on phytochemical pesticides. Boca Raton, Flórida: CRC,1989. p. 47-86.

13 SAUCKE, V. H.; SCHMUTTERER, H. Untersuchungen u"ber Nebenwirkungen verschiedener Niemprodukte bei Daphnia magna Strauss (Crustacea: Cladocera). Anzeiger Scha"dlingskunde Pflanzenschutz Umweltschutz, v. 65, n.7, p. 121-126, 1992.

14 STARK, J.D. Population-level effects on the nim insecticide Nimix on Daphnia pulex. Journal of Environmental Science and Health, Part B, v. 36, n.4, p. 457-465, 2001.

15 SUTTON, T. Environmental effects of nim-based insectisides on zooplankton communities in Forest ponds, 2002. p.19. Disponível em: <www.fnr.purdue.edu>. Acesso em: 26/09/2010.

\section{AGRADECIMENTOS}

Agradecemos à Fundação de Amparo à Pesquisa do Estado de São Paulo (FAPESP) pelo apoio financeiro. 